\title{
Propiedades psicométricas del instrumento Autopercepción del Estado
} Emocional (APEEM)

Psychometric properties of the Emotional State Self-Perception instrument (APEEM) Recepción del artículo: 07-11-19 | Aceptación del artículo: 02-12-19

Piñeyro Diego* diegopiney@gmail.com Azzollini Susana* susana1060@yahoo.com.ar Simkin Hugo * hugosimkin@psi.uba.ar Herrera Angela María** anmahelaherreraalvarez@gmail.com

Facultad del Ejército, Universidad de la Defensa

Nacional/ Facultad de Psicología, Universidad de Buenos Aires (Argentina)* Unidad Nacional de Educación Enrique Guzmán y Valle**

ORCID: https://orcid.org/* https://orcid.org/ 0000-00023192-5087* https://orcid.org/ 0000-0001$7162-146 X^{*}$ https://orcid.org/ 0000-00026399-3850**

Para referenciar este artículo:

Piñeyro, D., Azzollini, S., Simkin, H. y Herrera, A.M. (2019). Propiedades psicométricas del instrumento

Autopercepción del Estado Emocional (APEEM) Revista ConCiencia EPG, 4(2), 55-69. https://doi.org/10.32654/ CONCIENCIAEPG.4-2.5

\section{Resumen}

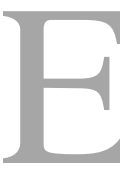

xisten diferentes técnicas para evaluar los niveles de estrés y ansiedad que una persona puede experimentar en un momento determinado (Olivares Rodríguez \& Mendez Carrillo, 1997; Spielberger, Gorsuch, \& Lushene, 2008). Sin embargo, son escasos o inexistentes los instrumentos que evalúan el estado de perturbación emocional (Shapiro, 2004) provocado por el recuerdo de una vivencia estresante, que cuenten con una adecuada validez y confiablidad. En el presente estudio se busca establecer las propiedades psicométricas de la técnica "Autopercepción del Estado Emocional" (Piñeyro \& Azzollini, 2016; Piñeyro, 2018), la cual evalúa el impacto emocional generado por el recuerdo específico de una vivencia estresante reciente. Se evaluó una población compuesta por 81 rescatistas peruanos y bolivianos, en los días inmediatos a la ocurrencia de un desastre natural. Se realizó un Análisis de Componentes Principales y un posterior Análisis Confirmatorio. Los resultados indican que el instrumento, para su escala resumida del factor perturbación emocional, posee una estructura compuesta por 2 dimensiones: Reactividad Ansiosa Predominantemente Cognitiva y Reactividad Ansiosa Predominantemente Fisiológica, las cuales explican el $73.6 \%$ de la varianza, con una validez de constructo cuyo modelo se ajusta satisfactoriamente (Chi-cuadrado X2 $=12.0034 ; 13 \mathrm{gl} ; \mathrm{p}=$ 0.527; $\chi^{2} / g l=0.923 ; R M S E A=0.000$, IC 90\% [0.000, 0.103]; $C F I=0.999 ; N F I=0.966 ; N N F I=0.999)$, y una muy buena fiabilidad por consistencia interna (Alfa de Cronbach $=0.88$ ).

Palabras clave: Impacto emocional, autopercepción del estado emocional, reactividad ansiosa, rescatistas de catástrofe ambientales. 
Abstract

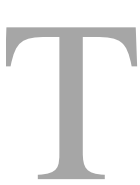

here are different techniques to assess the levels of stress and anxiety that a person may experience in specific circumstances (Olivares Rodríguez \& Mendez Carrillo, 1997; Spielberger, Gorsuch, \& Lushene, 2008). However, the instruments that estimate the state of emotional disturbance (Shapiro, 2004) are scarce or non-existent. This study seeks to establish the psychometric properties of the "Self-perception of the Emotional State" technique (Piñeyro \& Azzollini, 2016; Piñeyro, 2018), which assesses the emotional impact generated by the specific memory of a recent stressful experience. A population composed of 81 Peruvian and Bolivian rescuers was evaluated in the days immediately after the occurrence of a natural disaster. A Principal Components Analysis and a subsequent Confirmatory Analysis were performed. The results indicate that the instrument, for its summary scale of the emotional disturbance factor, has a structure composed of 2 dimensions: Predominantly Cognitive Anxious Reactivity and Predominantly Physiological Anxious Reactivity, which explain $73.6 \%$ of the variance, with a construct validity whose model fits satisfactorily (Chi-square $\chi^{2}=$ $12.0034 ; 13 \mathrm{gl} ; \mathrm{p}=0.527 ; \chi^{2} / \mathrm{gl}=0.923$; RMSEA $=0.000$, IC 90\% [0.000, 0.103]; $\mathrm{CFI}=0.999 ; \mathrm{NFI}=0.966 ; \mathrm{NNFI}=0.999)$, and very good reliability (Cronbach's alpha $=0.88$ ).

Keywords: emotional impact, selfperception of emotional state, anxious reactivity, environmental catastrophe rescuers.

\section{Introducción}

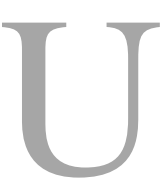

na serie de estudios realizados en rescatistas y personal de emergencias encargado de asistir a víctimas de catástrofes ambientales, indican que existe una mayor proporción de reacciones psicológicas peritraumáticas adversas en los mismos, con una prevalencia que supera a la encontrada en la población general, debido a las altas exposiciones a situaciones traumáticas que experimentan por sus labores (González Ordi, Miguel-Tobal, Cano Vindel, Iruarrizaga, 2004). Cerca del $35 \%$ de esta población experimenta síntomas de ansiedad que cumplen con los criterios de ataque de pánico según el DSM IV: Diagnostic and Statistical Manual of Mental Disorders (American Psychiatric Association, 1994).

La ansiedad se define como una reacción emocional normal, necesaria para la supervivencia, que en algunas personas puede alcanzar niveles patológicos, conduciendo a un trastorno de ansiedad cuando no se maneja adecuadamente, pudiendo alcanzar niveles excesivamente altos y poco adaptativos que afectan el ánimo y los aspectos funcionales (APA, 1994; Olivares Rodríguez et al., 1997, Spielberger et al., 2008). La falta de apoyo psicológico temprano en el personal de emergencias, justo cuando comienzan a experimentarse los primeros síntomas de ansiedad, puede conducir a un agravamiento de la sintomatología (Páez, Arroyo \& Fernández, 1995), consolidándose el malestar bajo la forma de diversos trastornos de ansiedad y el ánimo (trastornos del sueño, fobias, ataques de pánico, estrés postraumático, depresión, entre otros). La falta de tratamiento aumenta drásticamente las posibilidades de comorbilidad, pudiendo en cuestión de meses empeorar el cuadro de salud por la incorporación de nuevos trastornos. 
De esta manera, resulta frecuente la observación de casos de sujetos que comienzan con problemas para conciliar el sueño, para luego (en cuestión de días o semanas) sufrir de ataques de pánico, los cuales, al no ser tratados adecuadamente, pueden conducir a una agorafobia que no le permita a la persona salir de su domicilio sin acompañamiento, pudiendo culminar esta cadena de malestares en un trastorno de depresión mayor (Caballo, 1997; Olivares et al., 1997; Piñeyro \& Azzollini, 2015). Aunque la terapia cognitivo-conductual presenta diversas herramientas que resultan eficaces para los problemas de ansiedad, se necesitan nuevos procedimientos que mejoren la extensión y la duración de la respuesta al tratamiento, apuntando a reducir la vulnerabilidad al estrés vital, reforzando la percepción de autoeficacia y control personal (Caballo \& Vilchez, 2000). Más notoria se hace esta necesidad cuando consideramos la salud mental de poblaciones específicas, como es el caso del personal de emergencias, donde el desarrollo de tratamientos con eficacia terapéutica probada experimentalmente es escaso.

A través de intervenciones inmediatas, focalizadas y breves, diversas líneas de investigación intentan brindar una solución a esta problemática; por ejemplo, el protocolo de primera ayuda psicológica (PAP) para sobrevivientes de desastres naturales o antrópicos (Farchi, 2014; Piñeyro et al., 2015; Azzollini, Azzara, Depaula, Cosentino \& Grinhauz, 2017), propone herramientas de intervención sencillas y de aplicación individual que pueden ser utilizadas por cualquier persona con una mínima formación, facilitando en cuestión de minutos que la persona asistida ponga en funcionamiento las regiones cerebrales ligadas a la toma de decisiones (i.e. la corteza pre-frontal), inhibiendo el funcionamiento de otras áreas más ligadas al procesamiento emocional (i.e. la amígdala). Este cambio en el procesamiento cognitivo prevendría la fijación de huellas traumáticas, aprendizajes disfuncionales sustentados en engramas específicos (Lashley, 1950) que podrían posteriormente desencadenar en estrés postraumático. Otra propuesta, que intenta preservar el estado de salud mental del personal de emergencias, es la técnica PASSER (Piñeyro, Squilace, Depaula \& Cosentino, 2017), diseñada para el procesamiento asistido de situaciones estresantes recientes, a través de la cual profesionales de la salud pueden brindar una primera ayuda psicológica de forma grupal a rescatistas y personal de emergencias en general. Esta técnica brinda un andamiaje externo de recursos para la memoria de trabajo, el cual facilita y re-direcciona adaptativamente los procesos de recuperación, codificación y almacenamiento de las memorias explícitas e implícitas ligadas al evento estresante. Más allá de que ambas herramientas de intervención resultan prometedoras, las dificultades coyunturales de la aplicación de las mismas (lugar donde ocurre el desastre, hospital de campaña, centro de evacuados, cuartel de rescatistas) dificultan la utilización de diseños experimentales que permitan determinar la eficacia terapéutica y sus mecanismos subyacentes. Estas limitaciones para acceder a las poblaciones de estudio en ámbitos que permitan un buen control de variables contaminadoras, en circunstancias temporales que garanticen la validez ecológica del diseño, sumadas a la falta de predisposición de los sujetos a colaborar en tareas de investigación (debido al cansancio y al estrés que se viven durante una situación de emergencia), convierten toda iniciativa de rigor experimental en un verdadero desafío.

Si bien podemos encontrar algunas técnicas que permiten evaluar el estado de ansiedad o perturbación emocional que puntualmente experimenta una persona, como el inventario STAI (Spielberger et al., 2008; Fonseca-Pedrero, Paino, Sierra- 
Baigrie, Lemos-Giráldez \& Muñiz, 2012) o la escala de unidades subjetivas de perturbación (USP) de Shapiro (2004), las mismas no resultan del todo adecuadas para ser utilizadas en este ámbito específico, si se tienen en cuenta las particularidades anteriormente mencionadas. En este sentido, si consideramos la medición de USP, vemos que en la misma simplemente se cuantifica en una sola pregunta, en la que se solicita a la persona que valore la intensidad del malestar experimentado en una escala del 0 al 10 considerando las emociones vinculadas a una imagen traumática, registrando la zona corporal en que el malestar aparece (Shapiro, 2004; Lago Blanco \& Larraz Geijo, 2012). $\mathrm{Si}$ bien esta medición es ampliamente utilizada con fines terapéuticos y de investigación, su uso como operacionalización del impacto emocional de un recuerdo puede resultar inadecuada, ya que evalúa el fenómeno de manera muy general y carece de estudios adecuados de validez y confiabilidad. A su vez, es de esperar que esta técnica tenga una alta tasa de error de medición (ya que se utiliza un único reactivo), por lo que resulta necesario elaborar una nueva operacionalización que reduzca la varianza de error de la medición, resultando conveniente generar un cuestionario que permita sumar las puntuaciones de distintos reactivos, de forma tal que la cuota de error que tiene cada uno de ellos tienda a compensarse en las escalas sumadas que finalmente se obtengan (Batista Foguet, Coenders \& Alonso, 2004). Por otro lado, si se evalúa utilizando la dimensión "ansiedad estado" del STAI, solo se puede evaluar el nivel de ansiedad actual (y transitorio) de los sujetos, sin poder vincular fehacientemente los montos experimentados en relación con un evento o situación particular. De esta manera, se dificulta discriminar la ansiedad experimentada, que se deriva de las vivencias de las tareas de asistencia, de otras posibles fuentes de ansiedad, que intervienen como variables contaminadoras.
Ante esta problemática, la técnica "APEEM", de Autopercepción del Estado Emocional (Piñeyro et al., 2016, 2018), se presenta como una alternativa de abordaje. Su diseño sortea la mayor parte de los inconvenientes mencionados y alcanza una buena consistencia interna (Alpha de Cronbach $=0.8651$ ) en sus estudios preliminares, destacándose su forma de aplicación grupal por ser muy breve, simple de entender y contestar, siendo su característica distintiva su capacidad para evaluar el impacto emocional provocado por el recuerdo de una vivencia reciente, estresante y específica, que el personal de emergencias experimenta inmediatamente después de una jornada laboral, en circunstancias de asistencia en desastres (Piñeyro et al., 2016, 2018). Esta técnica fue diseñada para ser autoadministrada de forma rápida y económica por el personal de emergencias, facilitando su aplicación en contextos complejos como centros de evacuados, hospitales de campaña y cuarteles de rescatistas al final de una jornada de trabajo. De esta manera, se puede identificar tempranamente a aquellos sujetos que presentan dificultades para la elaboración de las vivencias estresantes, posibilitando también el diseño y ejecución de investigaciones experimentales en el área.

Nos proponemos evaluar las propiedades psicométricas de la técnica APEEM, a través de un análisis factorial exploratorio (AFE), con extracción de sus componentes principales (ACP), complementando el mismo con un análisis confirmatorio (AC).

\section{Método}

Diseño. - Se realizó un análisis factorial exploratorio para extraer los componentes principales y posteriormente un análisis confirmatorio del modelo.

Dado que la técnica APPEM fue diseñada principalmente para evaluar el 
impacto emocional que presentan rescatistas y personal de emergencias sobre situaciones estresantes recientes (derivado de su trabajo en situaciones de desastres naturales), se evaluó únicamente a aquellos que recientemente (dentro de los cuatro meses de ocurrencia) participaron en tareas de asistencia a víctimas en algún tipo de episodio considerado como emergencia o desastre natural. Con el objeto de controlar la varianza de error que podría provocar la evaluación de recuerdos de vivencias cualitativa y temporalmente diferentes, se homogeneizó experimentalmente la vivencia (y su evocación) y el tiempo transcurrido desde su ocurrencia hasta su medición, evaluando el impacto emocional del recuerdo del contenido de un video documental sobre un desastre natural (terremoto), el cual fue proyectado previamente a la toma de datos.

Participantes. - Se trabajó con una muestra de 81 rescatistas y personal de emergencias en general, quienes participaron voluntariamente de la investigación. Con una edad promedio de 29 años $(D E=8.86)$, un $60.49 \%$ de hombres y un 39.51 de mujeres, el grupo quedó conformado por; 42 sujetos de nacionalidad peruana, residentes de la comunidad de Lurigancho-Chosica (Lima), quienes fueron evaluados cuatro meses después de que la región sufriera uno de los peores desastres naturales de su historia, un huaico que arrasó con varias viviendas y dejó un saldo superior a las 120 víctimas fatales; y 39 bolivianos de la localidad de Tarija y Sucre, quienes fueron evaluados en las dos semanas posteriores a un importante incendio forestal en el que se registraron cuantiosas pérdidas materiales, con más de 7000 hectáreas de bosque perdidas, y un saldo de 3 rescatistas fallecidos durante las tareas de asistencia.

Instrumentos. - Los sujetos fueron evaluados con la técnica Autopercepción del Estado Emocional (APEEM), la cual fue utilizada de manera grupal (auto-administrada). La misma fue diseñada para evaluar el estado emocional que específicamente provoca el recuerdo de un suceso altamente estresante, induciendo, a través de sus instrucciones, a que los sujetos re-experimenten lo más vívidamente posible el recuerdo de una vivencia particular. Este instrumento está conformado por 15 ítems, de los cuales los primeros 9 valoran el estado emocional en una escala presentada gráficamente, en forma de barra continua, con valores discretos comprendidos entre el 0 y el 10, el resto de los ítems utilizan diferentes opciones de escala tipo Likert siendo 1 "completamente en desacuerdo" y 5 "completamente de acuerdo" (Piñeyro, et al., 2016, 2018).

Procedimiento. - Los sujetos fueron reclutados voluntariamente para participar de una investigación sobre aspectos psicológicos relacionados con las tareas de asistencia en desastres naturales. Los mismos fueron informados sobre el anonimato y la confidencialidad de sus respuestas, requiriéndoseles la firma de un consentimiento informado para la participación. La evaluación se realizó grupalmente en un salón adecuadamente equipado para la utilización de audio y video, con cómodos asientos individuales. Una vez instalados, se les informó que se proyectaría un video documental de 20 minutos de duración, el cual podría interesarles por estar relacionado con sus labores, dado que muestra imágenes de un terremoto de $7.8^{\circ}$, ocurrido el 16 de abril de 2016 en las costas de Ecuador, con testimonios reales de rescatistas y sobrevivientes, combinados con representaciones sobre lo sucedido llevadas adelante por actores.

El video documental fue realizado por la producción del programa Crónicas del Sábado del Canal 23 de Ecuador, el mismo fue editado para lograr una versión resumida 
a 20 minutos de los 41 de duración original, resaltando los aspectos que se consideraron más relevantes, eligiéndose aquellas escenas que por empatía podrían generar activación emocional en la población de estudio.

Una vez finalizada la proyección del video se repartieron las hojas con el inventario APPEM, solicitándoles a los sujetos que en silencio lean atentamente las instrucciones, y se tomen el tiempo necesario para seguirlas al pie de la letra.

\section{Resultados}

Se realizaron análisis descriptivos y un análisis factorial exploratorio de componentes principales utilizando el software SPSS, versión 11.0. Posteriormente se examinó la estructura interna con un análisis confirmatorio de ecuaciones estructurales, sometiendo a prueba el modelo hipotético mediante la técnica de Máxima Verosimilitud, utilizando el software EQS, versión 6.3 para Windows. Se utilizó el método robusto de máxima verosimilitud con la corrección de Satorra y Bentler. Se analizó el ajuste considerando el cociente $\chi^{2} / \mathrm{gl}$, el error cuadrático medio de aproximación (RMSEA) y los índices de ajuste comparativo (CFI), normalizado (NFI) y no normalizado (NNFI) considerando como valores referenciales adecuados para el ajuste: $\chi^{2} / \mathrm{gl} \leq 3$, RMSEA $\leq 0.06, \mathrm{CFI} \geq 0.95, \mathrm{NFI} \geq 0.95$ y NNFI $\geq$ 0.95 (Hooper, Coughlan \& Mullen, 2008; Batista-Foguet et al., 2004).

De los 15 ítems originales que conforman el instrumento se efectuó una primera depuración en función de la adecuación de la escala de medición, conservando únicamente los primeros 9 reactivos, los cuales valoran el estado emocional en una escala presentada gráficamente, en forma de barra continua, con valores discretos comprendidos entre el 0 (ausencia de emoción) y el 10 (máximo nivel de emoción experimentada), el resto de los ítems se descartaron por resultar inadecuados para los análisis pertinentes, debido a que miden en escalas nominales $u$ ordinales tipo Likert con menos de 3 opciones de respuesta, que no presentan una distribución normal. Esta restricción se debe a que los análisis de datos que proponen los distintos tipos de software utilizados analizan matrices de correlaciones o de covarianzas, para las cuales los ítems tienen que ser variables continuas, y cuando no es así, como suele ocurrir en la mayoría de los casos en test de las ciencias sociales que utilizan escalas tipo Likert, el requisito es que se acerquen al supuesto de continuidad, utilizando no menos de cinco alternativas de respuesta, con una distribución que se aproxime a la normalidad (Llorent Segura, Ferreres- Taver, Hernández- Baeza \& Tómas-Marco, 2014). Posteriormente se efectuó un análisis de validez de contenido de los 9 ítems seleccionados, descartando para los posteriores análisis a dos de ellos; "Grado de confianza en si mismo" y "Sensación de aislamiento o soledad que siente ahora", los cuales si bien permiten evaluar un aspecto relevante del estado emocional de la persona, contaminan la medición en cuanto a la reactividad emocional provocada por el recuerdo, ya que la medición de los mismos hace referencia a aspectos más ligados a rasgos estables de la persona y no tanto a una respuesta transitoria que es causada por los contenidos del recuerdo.

\section{Análisis Factorial de Componentes Principales}

Se efectuó un Análisis de Componentes Principales (ACP) sobre una muestra de 81 rescatistas y personal de emergencias en general, de nacionalidad peruana y boliviana. Se evaluó con el inventario APEEM en relación al recuerdo de un video documental proyectado, sobre un desastre natural (terremoto) ocurrido en Ecuador que cuenta con el testimonio de 
algunos sobrevivientes que fueron rescatados después de tres días de estar atrapados bajo los escombros de un shopping. Con este análisis se buscó extraer un número menor de factores que expliquen la mayor parte de la varianza de la muestra (Llorente Segura et al., 2014).

Como primer paso se realizó un análisis de la relación entre las variables con el test KMO de Kaiser, Meyer y Olkin (ver tabla 1) el valor encontrado de 0,838 indica que la relación entre los factores es alta (test muy bueno), lo que indica que el modelo factorial (o la extracción de sus factores) para estos datos es pertinente. De igual manera, el test de Esfericidad de Bartlett (ver tabla 1), el cual evalúa la aplicabilidad del análisis factorial de las variables estudiadas, arrojó valores muy significativos ( $p$ $<0.000)$, lo que indica que la matriz de datos es adecuada para el análisis.

Tabla 1

Prueba de Kaiser - Meyer - Olkin (KMO) y Bartlett

\begin{tabular}{ll} 
Kaiser-Meyer - Olkin Measure of Sampling Adequacy &, 838 \\
Bartlett`s Test of Sphericity Approx. Chi-Square & 355,435 \\
df & 21 \\
Sig. &, 000 \\
\hline
\end{tabular}

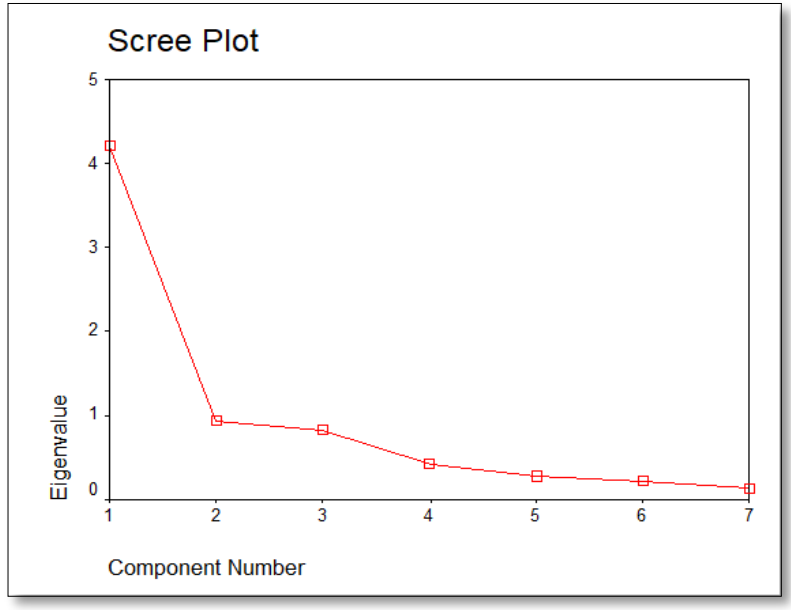

Figura 1. Scree Plot

Se decidió incluir dos componentes en el modelo, decisión que se alcanzó a través de una combinación de análisis que incluyen; el criterio de Kaiser, el análisis del gráfico de sedimentación, la teoría de la que se parte y la interpretabilidad de los datos encontrados. Si bien el criterio usado con mayor frecuencia por la comunidad científica es el de Kaiser, el cual propone incorporar factores con autovalores superiores a 1, el uso de este único criterio puede traer algunos inconvenientes (Llorente Segura et al., 2014). Por tal motivo se complementó la regla de Kaiser con el análisis de un gráfico de sedimentación (Scree Plot), a través del cual se pudo corroborar la adecuación de extraer dos factores mediante la observación de los elementos incluidos hasta el cambio brusco en la pendiente graficada, decisión que es consistente con la teoría de la que se parte y facilita la interpretación de los datos. De esta 
manera se decidió incorporar un segundo componente, flexibilizando la regla de Kaiser, con un autovalor de 0.938 (muy cercano a 1 aunque no lo supera). Con ambos componentes se logra un modelo que explica el $73.36 \%$ de la varianza total, porcentaje que es muy adecuado ya que supera ampliamente el límite requerido para publicaciones en ciencias sociales (superior al 50\%).

Tabla 2

Total Variance Explained

\begin{tabular}{|c|c|c|c|c|c|c|c|c|c|}
\hline \multirow{2}{*}{$\begin{array}{l}\text { Com } \\
\text { pone } \\
\text { nt }\end{array}$} & \multicolumn{3}{|c|}{ Initial Eigenvalues } & \multicolumn{3}{|c|}{$\begin{array}{l}\text { Extration Sums of Squared } \\
\text { Loadings }\end{array}$} & \multicolumn{3}{|c|}{$\begin{array}{l}\text { Rotation Sums oof Squared } \\
\text { Loadings }\end{array}$} \\
\hline & Total & $\begin{array}{l}\% \text { of } \\
\text { Varien } \\
\text { ce }\end{array}$ & $\begin{array}{l}\text { Cumulat } \\
\text { ive } \%\end{array}$ & Total & $\begin{array}{l}\% \text { of } \\
\text { Varience }\end{array}$ & $\begin{array}{l}\text { Cumul } \\
\text { ative } \\
\%\end{array}$ & Total & $\begin{array}{l}\% \text { of } \\
\text { Varience }\end{array}$ & $\begin{array}{l}\text { Cumul } \\
\text { ative } \\
\%\end{array}$ \\
\hline 1 & 4,217 & 60,236 & 60,237 & 4,217 & 60,236 & 60,236 & 2,826 & 40,365 & 40,365 \\
\hline 2 & ,938 & 13,400 & 73,636 & ,938 & 13,400 & 73,636 & 2,329 & 33,272 & 73,636 \\
\hline 3 & 821 & 11,725 & 85,362 & & & & & & \\
\hline 4 & ,417 & 5,954 & 91,315 & & & & & & \\
\hline 5 & ,267 & 3,818 & 95,133 & & & & & & \\
\hline 6 &, 214 & 3,060 & 98,194 & & & & & & \\
\hline 7 & ,126 & 1,806 & 100,000 & & & & & & \\
\hline
\end{tabular}

Extration Method: Principal Component Analysis

Se utilizó el método de rotación ortogonal Varimax (ver tabla 3). Para una mejor apreciación de los resultados se

Table 3

Rotated Component Matrix eliminaron las cargas factoriales inferiores a 0.40 en la impresión de la tabla de resultados. 
De los datos obtenidos se puede inferir que el constructo "impacto $o$ perturbación emocional”, provocado por el recuerdo de una vivencia altamente estresante, presenta dos dimensiones $\mathrm{o}$ componentes. La primera, que agrupa los 4 ítems: "Nivel de preocupación que ahora siente", "Nivel de inquietud y nerviosismo que ahora siente", "Nivel de tristeza que ahora siente" y "Nivel de miedo, sensación de peligro que ahora siente", resulta indicativa (por la composición de sus reactivos) de lo que podríamos llamar Reactividad Ansiosa Predominantemente Cognitiva. La segunda, que agrupa los 3 ítems: "Nivel de cansancio o fatiga que siente ahora", "Nivel de tensión muscular que siente ahora", "Nivel de ira o bronca que ahora siente", resulta indicativa de lo que podríamos llamar Reactividad Ansiosa Predominantemente Fisiológica.

\section{Análisis de Confiabilidad}

Se analizó la consistencia interna del instrumento considerando los 7 ítems seleccionados sobre la muestra de 81 casos. Se obtuvo un Alfa de Cronbach $=0.88$, lo que indica una muy buena consistencia interna, sobre todo si se considera el breve número de ítems del instrumento (Cronbach, 1951; Batista-Foguet et al., 2004).

\section{Análisis Factorial Confirmatorio}

Dado que en ACP los componentes extraídos funcionan como compuestos de las variables observadas, que solo cumplen la misión de reproducir al máximo la varianza de cada ítem (variable observada) en un mínimo número de compuestos, se complementó el estudio con un Análisis Factorial Confirmatorio (AFC), ya que para el primer método de análisis ni los componentes serían variables latentes, ni los ítems serían ninguna medida indirecta de ellos. A su vez el ACP supone ignorar el error de medida, lo que aumenta espuriamente las cargas factoriales, los porcentajes de varianza explicados por los factores, y puede producir una sobreestimación de la dimensionalidad (Llorent Segura, et. al, 2014). El ACP permite obtener una solución factorial inicial no restrictiva, la que posteriormente, mediante una AFC, puede ser transformada aplicando diferentes criterios de rotación para poner a prueba una solución única, cuya adecuación puede ser evaluada utilizando diferentes índices de bondad de ajuste (Ferrando \& Anguiano-Carrasco, 2010).

Partiendo de una fundamentación teórica del constructo, se eliminaron los ítems que podían afectar la validez de contenido y se establecieron dos dimensiones concordantes con los 2 componentes extraídos por el ACP. El modelo esbozado fue puesto a prueba con un AFC, para el cual se utilizó el software EQS versión 6.3 (Windows). El modelo propuesto se sintetiza en la siguiente figura. 


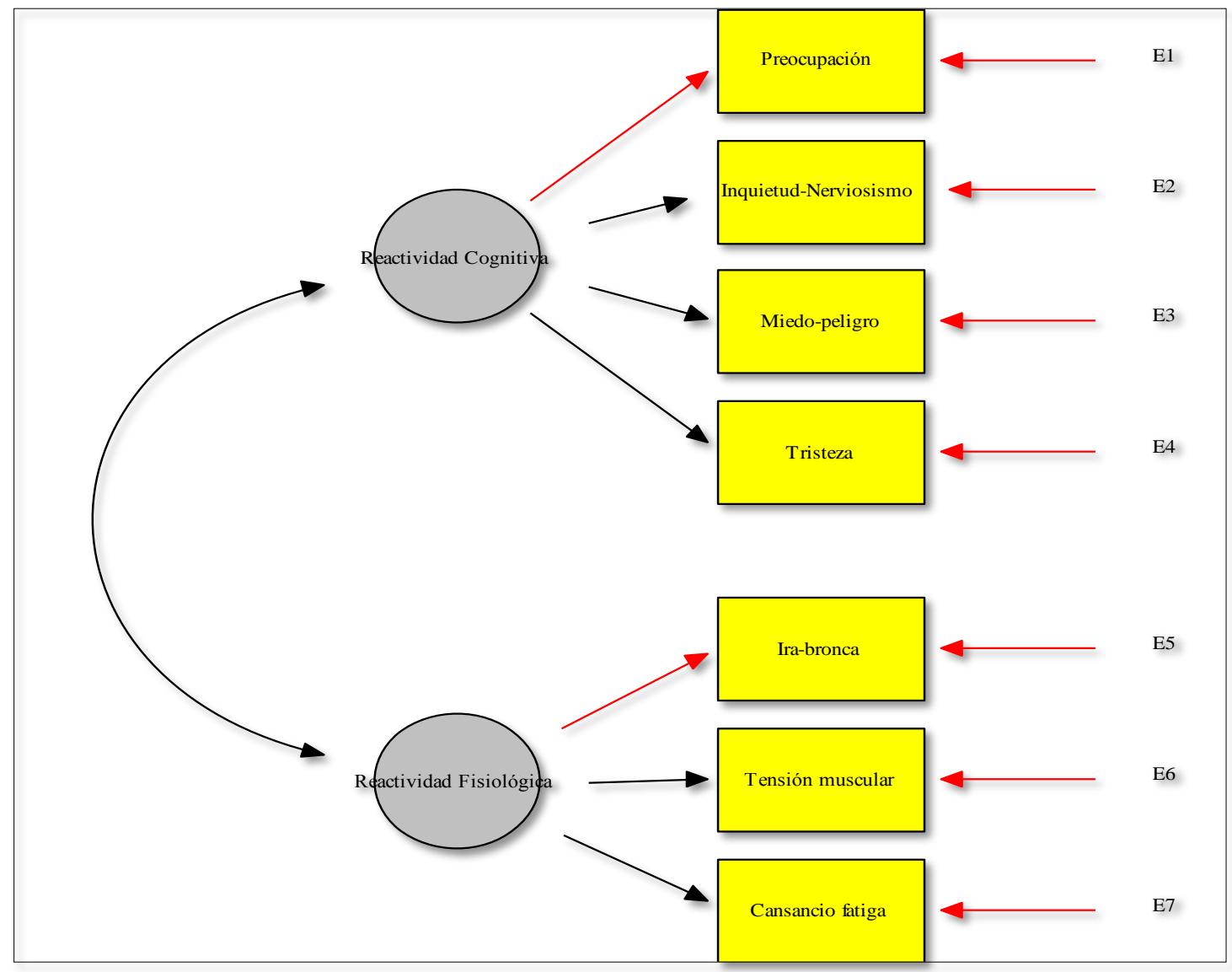

Figura 2. Análisis factorial confirmatorio

Donde el constructo impacto o perturbación emocional presenta dos dimensiones: Reactividad Ansiosa Predominantemente Cognitiva, que agrupa las variables "Nivel de preocupación que ahora siente", "Nivel de inquietud y nerviosismo que ahora siente", "Nivel de tristeza que ahora siente" y "Nivel de miedo, sensación de peligro que ahora siente"; y Reactividad Ansiosa Predominantemente Fisiológica, que agrupa "Nivel de cansancio o fatiga que siente ahora", "Nivel de tensión muscular que siente ahora" y "Nivel de ira o bronca que ahora siente".

Para validar el modelo se utilizó la técnica de Máxima Verosimilitud, Método Robusto, obteniéndose los siguientes índices de bondad de ajuste:

Satorra-bentler scaled chi-square $=$ $12.0034 ; 13 \mathrm{gl} ; p=0.527$
Este resultado indica que el modelo se ajusta adecuadamente a los datos analizados, su p valor superior a 0.05 $(p=0.527)$ indica que se debe sostener la hipótesis nula, la cual establece que los errores de nuestro modelo son nulos, al no resultar estadísticamente significativo $(\mathrm{p}>0.05)$ puede interpretarse como indicador de un adecuado ajuste del modelo a los datos. Así mismo el cociente obtenido de $\chi^{2} / g l=0.923$, lo cual se considera un buen indicador si el resultado oscila entre uno y tres o, de manera más flexible si el resultado es $\leq 5$ (Miranda-Zapata, Riquelme-Mella, Cifuentes-Cid \& Riquelme-Bravo, 2014). Y la Raíz cuadrada del error medio cuadrático $($ RMSEA $)=0.000$, con un $90 \%$ Confidence interval of RMSEA (0.000, 0.103). 
Como se puede observar el ajuste es muy bueno $(R M S E A=0.000)$ ya que valores inferiores a 0.08 son indicativos de buen ajuste, cumpliéndose con el requisito de que el intervalo de confianza incluya el valor cero. El Índice de ajuste Comparativo $(C F I)=0.999$ y los valores superiores a 0.95 indican un buen ajuste del modelo. Así mismo el Índice de ajuste Normalizado de Bentler-Bonett $(N F I)=0.966$ y el valor alcanzado (superior a 0.95 ) es indicativo de buen ajuste. El Índice de ajuste No Normalizado de Bentler-Bonett $(N N F I)=$ 0.999 .

Esté índice, también llamado de Tucker-Lewis (TLI), compara el ajuste por grados de libertad del modelo propuesto y nulo (modelo de ausencia de relación entre las variables). Cuando su valor supera 0.95 indica un muy buen ajuste.

El análisis global de los diferentes índices de bondad de ajuste obtenidos indica que los datos se ajustan muy satisfactoriamente al modelo propuesto (Batista-Foguet et al., 2004).

\section{Discusión}

El propósito de esta investigación fue evaluar las propiedades psicométricas del instrumento "Autopercepción del Estado Emocional" (Piñeyro et al., 2016, 2018), la cual evalúa el impacto emocional generado por el recuerdo específico de una vivencia estresante reciente. De esta manera, se examinó la estructura interna, la consistencia de las puntuaciones y su ajuste a un modelo bidimensional para su escala resumida, la cual resulta valiosa para el desarrollo de investigaciones en el área de la salud mental de rescatistas y personal de emergencias en general, por su sencillez y rapidez de autoaplicación, cualidades necesarias para poder llevar adelante investigaciones experimentales que garanticen la validez ecológica. Poder evaluar de manera cuantitativa, valida y confiable, el estado emocional de los rescatistas en los momentos posteriores a una situación de desastre natural permite recabar datos fiables para evaluar los impactos perjudiciales sobre la salud mental, y posibilita el diseño y la evaluación de intervenciones apropiadas, pudiendo establecer la eficacia de las mismas estadísticamente. Disponer de datos psicométricos en cuanto a validez y confiabilidad, que avalen y justifiquen la utilización del instrumento APEEM para una muestra y un contexto determinado, es un paso necesario en este sentido (Argibay, 2006).

A través de una revisión teórica y estadística se reformó el inventario original (Piñeyro et al., 2016) a una versión resumida (véase el Anexo), la cual mejora las posibilidades de aplicación en contextos de gran validez ecológica, pero de difícil evaluación, como son los centros de evacuados, hospitales de campaña, o cuarteles de rescatistas durante una jornada de trabajo.

El constructo impacto emocional presenta dos dimensiones; Reactividad Ansiosa Predominantemente Cognitiva, la cual agrupa los 4 ítems: "Nivel de preocupación que ahora siente", "Nivel de inquietud y nerviosismo que ahora siente", "Nivel de tristeza que ahora siente" y "Nivel de miedo, sensación de peligro que ahora siente" ; y Reactividad Ansiosa predominantemente Fisiológica, que agrupa los 3 ítems: "Nivel de cansancio o fatiga que siente ahora", "Nivel de tensión muscular que siente ahora", "Nivel de ira o bronca que ahora siente". De esta forma se alcanzan los requerimientos mínimos de 3 a 4 ítems por factor (Ferrando et al., 2010), eliminando los ítems redundantes que deterioran la estructura factorial resultante, ya que los reactivos con una redacción mínimamente distinta tienden a sobreestimar la consistencia interna de las escalas, incluso pudiendo generar factores comunes 
adicionales difíciles de explicar (Llorent Segura, et al., 2014; Batista-Foguet et al., 2004). Vale aclarar que, más allá de las restricciones de los análisis confirmatorios, que establecen que las variables que tienen carga sobre un factor presentan carga nula sobre los otros, debe considerarse con flexibilidad este aspecto, ya que esta situación restrictiva resulta muchas veces poco realista para factores que están correlacionados entre sí, donde la mayor parte de los ítems analizados no van a actuar como marcadores simples, ya que presentan cargas cruzadas menores que no son nulas, es decir que actúan sobre otros factores (Ferrando et al., 2010; Llorent Segura et al., 2014). De esta manera, resulta más adecuado distinguir las dimensiones o factores latentes como predominantemente Cognitivos, por presentar un mayor grado de introspección reflexiva y elaboración consciente, y predominantemente Fisiológicos, más determinadas por sensaciones corporales, sin perder de vista que estas cualidades no son totalmente exclusivas y excluyentes, y que se encuentran presentes en ambos factores (aunque en proporciones diferentes).

La escala reducida de APEEM presenta adecuadas propiedades psicométricas, su fiabilidad por consistencia interna resulta muy buena (Alfa de Cronbach $=0.88$ ), ya que supera cómodamente el límite de confiabilidad habitualmente requerido (0.70). $\mathrm{Su}$ estructura interna bidimensional explica el $73.6 \%$ de la varianza, con una validez de constructo cuyo modelo se ajusta satisfactoriamente (Chi-cuadrado X2= $12.0034 ; 13 g l ; p=0.527 ; \chi^{2} / g l=0.923$;

\section{Referencias}

Argibay, J.C. (2006). Técnicas psicométricas. Cuestiones de validez y confiabilidad. Subjetividad y Procesos Cognitivos, 8, 15-33.
RMSEA $=0.000$, IC 90\% [0.000, 0.103]; $C F I=0.999 ; N F I=0.966 ; N N F I=0.999)$.

El presente estudio debe considerarse contemplando las siguientes limitaciones; su muestra de 81 rescatistas, aunque resulta adecuada para la recomendación basada en la ratio personas /ítem $(\mathrm{N} / \mathrm{p})$, cuya requisito más habitual es la llamada "regla de los 10" (una muestra 10 veces mayor que el número de ítems que se evalúa) (Velicer \& Fava ,1998), o la proporción de 5 a 1 (Gorsuch,1993; Llorent Segura, et al., 2014), resulta escasa según recomendaciones más actuales que sugieren tener una muestra mínima de 200 sujetos (Ferrando et al., 2010; Llorent Segura, et al., 2014). En este sentido, podemos argumentar que, aunque es sabido que cuanto mayor la muestra más precisos son los análisis efectuados, considerando las dificultades de acceso a la muestra elegida para este tipo de estudio (personal de emergencia que intervino recientemente en una situación desastre natural), los casos evaluados se consideraron suficientes para evaluar y presentar los primeros resultados de la técnica, esperando que con futuras investigaciones se puedan corroborar estos resultados. Por otro lado, las características de la muestra (personal de emergencias de Bolivia y Perú) limitan la generalización de los resultados a otras poblaciones de interés. A su vez, no se controlaron variables que pueden resultar relevantes como la existencia de trastornos de ansiedad y otras patologías mentales que podrían modular las mediciones. Queda para futuras investigaciones profundizar sobre los propósitos de este estudio con las consideraciones pertinentes.

American Psychiatric Association. (1994). DSM IV- Diagnostic and Statistical Manual of Mental Disorders. APA. 
Azzollini, S.C., Azzara, S.H., Depaula, P.D., Cosentino, A.C., \& Grinhauz, A.S.

(2017). Modificación del protocolo de primera ayuda psicológica en situaciones de crisis. Acta Psiquiátrica y Psicológica de América Latina, 63 (2), 139-147. http://www.acta.org.ar/04-

WebForms/frmResumen.aspx? ?dArti culo $=1320$

Batista-Foguet, J.M., Coenders, G. y Alonso, J. (2004). Análisis factorial confirmatorio. Su utilidad en la validación de cuestionarios relacionados con la salud. Medicina Clínica, 122, 21-27. https://doi.org/10.1157/13057542.

Caballo, V. (1997). Manual para el tratamiento Cognitivo-Conductual de los trastornos psicológicos, 1. Trastornos por ansiedad, sexuales, afectivos y psicóticos. Siglo XXI.

Caballo V. \& Vilchez M. (2000) El tratamiento de los trastornos de ansiedad a las puertas del siglo XXI. Psicología Conductual, 8(2), 173215.

Cronbach, L. J. (1951). Coefficient alpha and the internal structure of de tests. Psychometrika, 16, 297- 334.

Farchi, M. (2014, agosto). Introducción a la Primera Ayuda Psicológica en situaciones de desastres y emergencias masivas. Seminario presentado en Crisis Management and Trauma Prevention in Disasters and Massive Emergencies, Buenos Aires, Argentina.

Ferrando, P. y Anguiano-Carrasco, C. (2010). El análisis factorial como técnica de investigación en psicología. Papeles del Psicológo,
31(1),

18-33.

https://www.redalyc.org/pdf/778/778 12441003.pdf.

Fonseca-Pedrero, E., Paino, M., SierraBaigrie, S., Lemos-Giráldez, S. y Muñiz, J. (2012). Propiedades psicométricas del cuestionario "Ansiedad Estado-Rasgo" (STAI) en universitarios. Behavioral Psychology / Psicología Conductual, 20 (3), 547561

González Ordi, H., Miguel-Tobal, J., Cano Vindel, A. y Iruarrizaga, I. (2004). Efectos de la exposición a eventos traumáticos en personal de emergencias: consecuencias psicopatológicas tras el atentado del 11-M. Ansiedad y Estrés, 10(2-3), 207-217

Gorsuch, R. L. (1983). Factor analysis (2nd. ed.). Erlbaum

Hooper, D., Coughlan, J., Mullen, M. (2008). Structural Equation Modelling: Guidelines for Determining Model Fit. Electronic Journal of Business Research Methods, 6(1), 53-60.

Lashley, K. S. (1950). In search of the engram. In Society for Experimental Biology, Physiological mechanisms in animal behavior. (Society's Symposium IV.) (pp. 454-482). Academic Press.

Lago Blanco,E. \& Larraz Geijo,G.(2012). EMDR: Revisión de la técnica y aplicación a un caso clínico. C. Med. Psicosom, 104, 45-56.

Llorent Segura, S., Ferreres- Taver, A., Hernández- Baeza, A. y TómasMarco, I. (2014). El análisis factorial exploratorio de los ítems: una guía práctica, revisada y actualizada. 
Anales de Psicología, 30(3), 11511169.

Miranda-Zapata, E., Riquelme-Mella, E., Cifuentes-Cid, H. \& Riquelme-Bravo, P. (2014) Análisis factorial confirmatorio de la Escala de habilidades sociales en universitarios chilenos. Revista Latinoamericana de Psicología, 46(2),73-82.

Shapiro, F. (2004). EMDR: Desensibilizacion y reprocesamiento por medio de movimiento ocular. Editorial Pax Mexico.

Spielberger, C. D., Gorsuch, R. L. y Lushene, R. E. (2008). STAI. Cuestionario de ansiedad estadorasgo ( $7^{\mathrm{a}}$ ed. rev.). TEA

Olivares Rodríguez, J. \& Mendez Carrillo F. (1997). Técnicas de Modificación de Conducta. Biblioteca Nueva.

Páez, D., Arroyo, E. y Fernández, I. (1995). Catástrofes, situaciones de riesgo y factores psicosociales. Mapfre Seguridad, 57, 43-55.

Piñeyro, D. y Azzollini, S. (2015) Intervención psicosocial en los planes de contingencia para catástrofes ambientales. En E. Fenoglio, M. Argerich, M. Peralta, N. Castillo, L. Di Pietro (Comp.). Inundaciones urbanas y cambio climático. Recomendaciones para la gestión (pp.125-128). Abrapalabra.

Piñeyro, D. \& Azzollini, S. (2016). Desarrollo del Instrumento APEEM, para la evaluación y medición del estado emocional del personal de emergencias que interviene en desastres ambientales. VIII Congreso Internacional de Investigación y Práctica Profesional en Psicología XXIII Jornadas de Investigación XII Encuentro de Investigadores en Psicología del MERCOSUR. Facultad de Psicología - Universidad de Buenos Aires, Buenos Aires. Tomo 2, pag.67

Piñeyro, D., Squilace, M., Depaula, P., Cosentino, A. (2017). Desarrollo de la Técnica PASER: Procesamiento Asistido para Situaciones Traumáticas Recientes en Rescatistas. Memorias del IX Congreso Internacional de Investigaciones $y$ Práctica Profesional en Psicología de la Facultad de Psicología de la Universidad de Buenos Aires. CABA: Facultad de Psicología de la UBA.

Piñeyro, D. (2018) Estudio psicométrico de la fiabilidad del instrumento APEEM (Autopercepción del Estado Emocional para la evaluación del estado emocional provocado por recuerdos estresantes. MEMORIAS X Congreso Internacional de Investigación y Práctica Profesional en Psicología. XXV Jornadas de Investigación. XIV Encuentro de Investigadores en Psicología del MERCOSUR), Tomo 1. pp 84-85.

Velicer, W. \& Fava, J. (1998). Effects of Variable and Subject Sampling on Factor Pattern Recovery. Psychological Methods. 3. 231-251. 


\section{Apéndice A}

APEEM: Autopercepción del estado emocional (Versión Breve) Piñeyro; Azzollini

Por favor siga las instrucciones paso por paso.

PASO 1: Tómese un momento para pensar sobre las situaciones que ha visto recientemente durante las tareas de asistencia a la población...

Intente recordar durante algunos segundos prestando atención a las imágenes, los sonidos, los olores y sensaciones que aparecen con el recuerdo. (Tómese su tiempo antes de continuar leyendo)

PASO 2: Tómese UN MINUTO para observar las imágenes de su recuerdo. ESTA VEZ CON LOS OJOS CERRADOS.

PASO 3: Responda las siguientes preguntas sobre las emociones y sensaciones que HOY, AQUÍ y AHORA siente. Indique (con una cruz) el nivel intensidad de las mismas en una escala del 0 al 10, considerando que el valor 0 (cero) es ausencia absoluta de la emoción/sensación evaluada mientras que 10 equivaldría al valor máximo de la misma.

Nivel de preocupación que ahora siente (marque con una cruz)

0 -1

Nivel de inquietud y nerviosismo que ahora siente

\begin{tabular}{|llllllllllll}
$\mathbf{0}$ & 1 & 2 & 3 & 4 & 5 & 6 & 7 & 8 & 9 & 10 \\
\hline
\end{tabular}

Nivel de miedo, sensación de peligro que ahora siente

\begin{tabular}{llllllllllll|l}
0 & 1 & 2 & 3 & 4 & 5 & 6 & 7 & 8 & 9 & 10
\end{tabular}

Nivel de tristeza que ahora siente

\begin{tabular}{|lllllllllll|l}
\hline 0 & 1 & 2 & 3 & 4 & 5 & 6 & 7 & 8 & 9 & 10 \\
\hline
\end{tabular}

Nivel de ira o bronca que ahora siente

\begin{tabular}{|llllllllllll|l}
$\mathbf{0}$ & 1 & 2 & 3 & 4 & 5 & 6 & 7 & 8 & 9 & 10 \\
\hline
\end{tabular}

Nivel de tensión muscular que siente ahora

\begin{tabular}{lllllllllll|l}
0 & 1 & 2 & 3 & 4 & 5 & 6 & 7 & 8 & 9 & 10
\end{tabular}

Nivel de cansancio o fatiga que siente ahora

\begin{tabular}{|llllllllllll|l}
$\mathbf{0}$ & 1 & 2 & 3 & 4 & 5 & 6 & 7 & 8 & 9 & 10 \\
\hline
\end{tabular}

